\title{
Budget Impact of Belantamab Mafodotin (Belamaf) Adoption in the Treatment of Patients with Relapsed or Refractory Multiple Myeloma in the United States
}

\author{
Anshul Shah' \\ Jonathan C Tosh ${ }^{2}$ \\ Apoorva Ambavane 2 \\ Andreas Nikolaou (1D) ${ }^{2}$ \\ Cosmina $\mathrm{Hogea}^{3}$ \\ Yevgeniy Samyshkin (iD) \\ Boris Gorsh ${ }^{3}$ \\ Eric M Maiese ${ }^{5}$ \\ Feng Wang ${ }^{3}$ \\ 'Modelling and Simulation, Evidera, \\ Waltham, MA, USA; ${ }^{2}$ Modelling and \\ Simulation, Evidera, London, UK; ${ }^{3}$ Value \\ Evidence and Outcomes, GlaxoSmithKline, \\ Upper Providence, PA, USA; ${ }^{4}$ Value Evidence \\ and Outcomes, GlaxoSmithKline, Brentford, \\ UK; ${ }^{5}$ Value Evidence and Outcomes, \\ GlaxoSmithKline, Philadelphia, PA, USA
}

Correspondence: Anshul Shah Evidera, 500 Totten Pond Road, Suite 500, Waltham, MA, 0245I, USA

Tel + I $781640894 \mid$

Email Anshul.shah@evidera.com
Purpose: Estimate the budget impact of belantamab mafodotin (belamaf) for patients with relapsed/refractory multiple myeloma (RRMM) who have received $\geq 4$ prior therapies, including an anti-CD38 monoclonal antibody, a proteasome inhibitor, and an immunomodulatory agent.

Methods: A budget impact analysis (BIA) was developed to estimate the cost difference between current (no belamaf) and projected (with belamaf) market scenarios over 3 years. Comparators were identified from a systematic literature review and included selinexor + dexamethasone or best supportive care. The number of treatment-eligible patients were estimated using an epidemiology model. Base-case analyses were conducted from a US commercial payer perspective (cost year: 2019). Model inputs included market share estimates, treatment duration, and costs of drug acquisition/administration, concomitant medications, adverse event (AE) management, treatment monitoring, and subsequent treatments based on published literature/cost databases. Budget impact, calculated as the difference in costs between current and projected scenarios over 3 years, was reported as cost per member per month (PMPM) and per member per year (PMPY). One-way sensitivity analysis assessed which key parameters most affected model outcomes. Alternative scenarios were tested (1or 5-year time horizon; Medicare perspective; negligible cost of mental status change AE).

Results: In a hypothetical commercial payer health plan with 1 million members, 33 patients were identified as treatment-eligible over 3 years. Introducing belamaf for patients with RRMM resulted in an estimated budget-neutral PMPM cost of $-\$ 0.0003$ and PMPY of $-\$ 0.004$, based on $n=9 / 33$ patients receiving treatment. Sensitivity analyses showed that budget impact in the base case was most sensitive to changes in treatment duration and drug acquisition costs. Base-case results were consistent across all scenarios assessed.

Conclusion: BIA indicates that adoption of belamaf in this patient population would be budget neutral for a US health plan.

Keywords: BCMA, belamaf, budget impact analysis, relapsed/refractory, multiple myeloma, payer

\section{Introduction}

Multiple myeloma (MM) is the third most common type of hematological cancer in the United States, accounting for $1.8 \%$ of all new cancer cases, with an estimated 140,779 patients living with the disease in 2017 and an estimated 32,270 new cases in $2020 .{ }^{1} \mathrm{MM}$ is responsible for $2.1 \%$ of all cancer deaths and the 5-year relative survival in 2010-2016 was 53.9\%. It is estimated that in 2020, MM was responsible for 12,830 deaths in the United States alone. ${ }^{1}$ 
Despite improvements in patient outcomes, such as increased response rate and progression-free and/or overall survival (OS) with the introduction of immunomodulatory agents, proteasome inhibitors (PI), and monoclonal antibodies $(\mathrm{mAb}), \mathrm{MM}$ remains incurable and almost all patients will eventually relapse and require several lines of therapy. ${ }^{2-4}$ Patients with relapsed/refractory MM (RRMM) have the highest unmet need, given their poor prognosis; with each subsequent relapse, there is a reduced depth of response, duration of remission, and survival rate. ${ }^{4,5}$

The National Comprehensive Cancer Network 2021 guidelines recommend several regimens for the management of RRMM, including bortezomib-, carfilzomib-, daratumumab-, ixazomib-, isatuximab-, pomalidomide-, panobinostat-, and elotuzumab-based regimens. ${ }^{6}$ Additional novel therapies were recently added to the treatment landscape for RRMM following the US Food and Drug Administration (FDA) approval of selinexor (a nuclear export inhibitor) in combination with dexamethasone (SEL+DEX) (July 2019) and single-agent belantamab mafodotin (belamaf [BLENREP]; a B-cell maturation antigen [BCMA]-directed antibody and microtubule inhibitor conjugate) for the treatment of RRMM in patients who have received $\geq 4$ prior therapies, including an anti-CD38 monoclonal antibody (mAb), a PI, and an immunomodulatory agent (August 2020). ${ }^{6-8}$

Belamaf is a humanized, afucosylated, antibody-drug conjugate targeting BCMA, which is highly expressed on MM cells, and eliminates myeloma cells by a multimodal mechanism. ${ }^{9-11}$ Single-agent belamaf demonstrated deep and durable responses in two clinical trials: the Phase I study DRiving Excellence in Approaches to Multiple Myeloma 1 (DREAMM-1 study; NCT02064387) and the pivotal Phase II study (DREAMM-2; NCT03525678) in heavily pretreated patients with RRMM. $^{12-14}$ The responses reported with belamaf $2.5 \mathrm{mg} / \mathrm{kg}$ once every 3 weeks in the primary analysis of the DREAMM-2 study (median follow-up: 6.3 months; overall response rate [ORR]: $31 \%)^{13}$ were sustained at 13 months; the ORR was $32 \%$ with an estimated median duration of response of 11.0 months, median OS of 13.7 months, and median progression-free survival (PFS) of 2.8 months. ${ }^{15}$

While clinical outcomes have improved with the introduction of newer drug therapies for RRMM in recent years, prolonged survival increases the likelihood of receiving multiple lines of therapy. ${ }^{16}$ Additionally, managing RRMM remains a high patient- and economiccost burden. ${ }^{16,17}$ Quantification of the potential budget impact of new therapies is needed so that payers are better informed to manage oncology-related costs. ${ }^{18,19}$ Budget impact analysis (BIA) evaluates the expected changes in the expenditure of a health payer after adoption of a new treatment and informs on the affordability of the new treatment. BIA is becoming an integral part of listings or submissions required by reimbursement authorities. ${ }^{20}$

The objective of this model-based BIA was to estimate the potential financial impact on US health plans of including belamaf for the treatment of patients with RRMM who have received $\geq 4$ prior therapies, including an anti-CD38 $\mathrm{mAb}$ antibody, a PI, and an immunomodulatory agent.

\section{Materials and Methods}

The budget impact model (BIM) was developed in accordance with the Academy of Managed Care Pharmacy and the International Society for Pharmacoeconomics and Outcomes Research Task Force on Good Research Practice guidelines. ${ }^{20,21}$

\section{Target Population}

The target population for this BIA was adult patients with RRMM who have received $\geq 4$ prior therapies, including an anti-CD38 mAb, a PI, and an immunomodulatory agent in accordance with the FDA-approved indication for belamaf.

\section{Treatments}

The BIM analysis considered only those treatment regimens used in US clinical practice ${ }^{6}$ and approved by the FDA for the target population. A systematic literature review (SLR) with a publication cut-off date of May 6, 2019, was performed to identify clinical trials comparable to the DREAMM-2 study (NCT03525678) of belamaf. The SLR was conducted using Embase, Medline, and MEDLINE inProcess, Cochrane Collection Central Register of Clinical Trials, the Database of Abstracts of Reviews of Effects, and Cochrane Database of Systematic Reviews and National Institute for Health Research-Health Technology Assessment. The SLR identified 40 publications with 22 studies representing standard of care $(\mathrm{SoC})$ for patients who have received $\geq 3$ prior lines of therapy (LOT). Of the 22 studies, 7 randomized clinical trials (RCTs), 8 single-arm trials, 4 observational studies, and 3 pooled analyses of RCTs were identified. Only STORM (NCT02336815) Part 2 was comparable with the DREAMM-2 study in terms of study 
design and baseline patient compatibility (adults with RRMM with $\geq 3$ prior lines of therapy; refractory to a PI and immunomodulatory agent and prior exposure to an antiCD38 mAb (Supplementary Figure S1)). ${ }^{13,22,23}$ The chosen comparators for belamaf were, therefore, SEL+DEX or best supportive care (BSC), which encompassed managing bone pain and anemia in patients with RRMM. ${ }^{24}$

\section{Model Perspective and Time Horizon}

The budget impact of belamaf was estimated from a US third-party commercial payer perspective for the base-case analysis, which employed a 3-year time horizon.

\section{Model Overview}

The BIM is estimated from a US third-party payer perspective and provides flexibility to conduct analysis from either a commercial or Medicare payer perspective. An overview of the BIM structure is shown in Figure 1. Costs were compared between the following scenarios to calculate the budget impact: (1) current market mix, a scenario with available treatment options for the target population prior to the reimbursement of belamaf, and (2) future market mix, a hypothetical scenario including belamaf in the treatment mix with a market uptake over the time horizon. Model inputs including market share data and sources are summarized in Table 1.

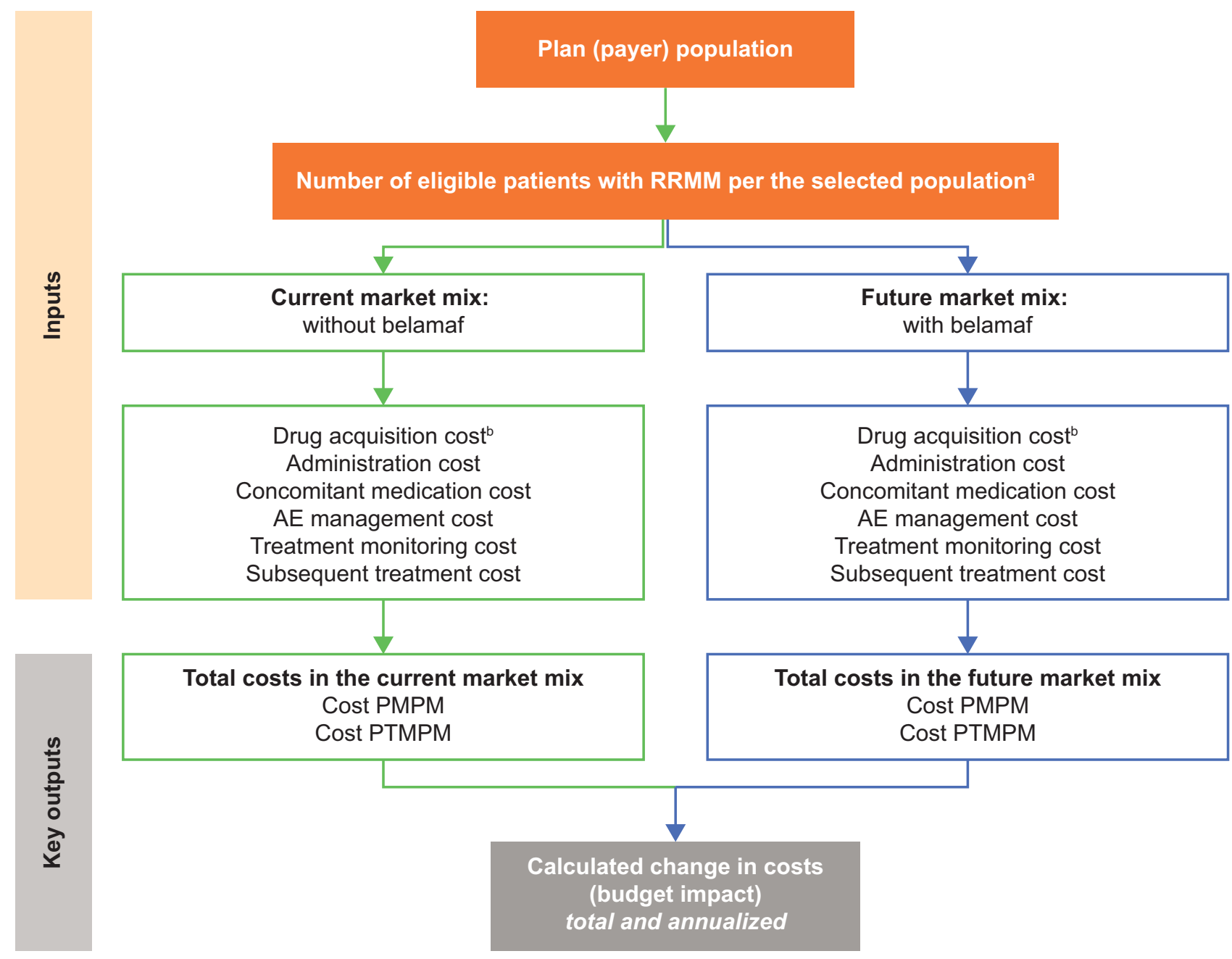

Figure I Model structure.

Notes: ${ }^{a}$ Selected population is adult RRMM patients who have received $\geq 4$ prior therapies including an anti-CD38 monoclonal antibody, a proteasome inhibitor, and an immunomodulatory agent; ${ }^{b}$ Includes flexibility to account for patient access schemes (user-modifiable input) and vial sharing option per US Food and Drug Administration label (user-modifiable input).

Abbreviations: AE, adverse event; belamaf, belantamab mafodotin; PMPM, per member per month; PTMPM, per treated member per month; RRMM, relapsed/refractory multiple myeloma. 
Table I Model Inputs

\begin{tabular}{|c|c|c|c|c|}
\hline \multirow[t]{2}{*}{ Input } & \multicolumn{3}{|l|}{ Details } & \multirow[t]{2}{*}{ Data Source(s) } \\
\hline & Belamaf & SEL+DEX & BSC Only & \\
\hline Patient characteristics & \multicolumn{3}{|c|}{ Mean (SD) body weight $78.4(21.80) \mathrm{kg}$} & DREAMM-2 $2^{13,25}$ \\
\hline Population and epidemiology ${ }^{\mathrm{a}}$ & \multicolumn{3}{|c|}{$\begin{array}{l}\text { Target population estimated using epidemiology model (full details of model reported } \\
\text { separately) }\end{array}$} & $\begin{array}{l}\text { Estimating the Number of US } \\
\text { Patients with Multiple Myeloma at } \\
5 \text { or More Lines of Treatment } \\
(\text { LOT })^{26}\end{array}$ \\
\hline Market share ${ }^{b}$ & $0 \%$ Current, $32 \%$ future & $64 \%$ Current, $32 \%$ future & $\begin{array}{l}36 \% \text { Current and } \\
\text { future }\end{array}$ & $\begin{array}{l}\text { Published BIM for SEL+DEX and } \\
\text { BSC only }{ }^{18}\end{array}$ \\
\hline Mean treatment duration & 2.99 months $^{c}$ & 2.99 months $^{c}$ & $\begin{array}{l}36 \text { months (full- } \\
\text { time horizon of } \\
\text { model) }\end{array}$ & $\begin{array}{l}\text { DREAMM-2 for belamaf } \\
\text { STORM for SEL+DEX }\end{array}$ \\
\hline Drug costs: Dosing and dose intensity & $\begin{array}{l}\text { Belamaf } 2.5 \mathrm{mg} / \mathrm{kg} ; 82.8 \% \\
\text { intensity; one administration } \\
\text { Q3W }\end{array}$ & $\begin{array}{l}\text { SEL: } 80 \text { mg; I00\% intensity; } 8 \\
\text { administrations Q4W } \\
\text { DEX: } 20 \text { mg; 100\% intensity; } 8 \\
\text { administrations Q4W }\end{array}$ & - & $\begin{array}{l}\text { Dosing from FDA label and clinical } \\
\text { trials } 7,8,13,22\end{array}$ \\
\hline Drug costs: Drug acquisition ${ }^{d}$ & $\begin{array}{l}\$ 16,859 \text { per cycle; } \\
\$ 24,435 \text { per month }\end{array}$ & $\begin{array}{l}\text { SEL: } \$ 22,000 \text { per cycle, } \\
\$ 23,915 \text { per month } \\
\text { DEX: } \$ 40 \text { per cycle, } \$ 43 \text { per } \\
\text { month } \\
\text { BSC only: } \$ 577 \text { per month }\end{array}$ & $\begin{array}{l}\text { BSC only: } \$ 577 \text { per } \\
\text { month }\end{array}$ & $\begin{array}{l}\text { Unit costs for SEL+DEX from Red } \\
\text { Book }^{\circledR} 2019^{31} \\
\text { Unit costs for belamaf from GSK } \\
\text { Unit costs for BSC only from } \\
\text { published BIM ( } \$ 567 \text { in } 2018 \text {, } \\
\text { inflated to } 2019 \text { value) }{ }^{18}\end{array}$ \\
\hline Administration costs & $\begin{array}{l}\text { 30-minute infusion, } \\
\text { administration cost } \$ 546 \text { per } \\
\text { administration and } \$ 791 \text { per } \\
\text { month }\end{array}$ & $\begin{array}{l}\text { Oral administration, no } \\
\text { administration costs }\end{array}$ & $\begin{array}{l}\text { No administration } \\
\text { costs }\end{array}$ & $\begin{array}{l}\text { Administration schedule from } \\
\text { FDA labels and clinical trials } \\
\text { Unit costs from inHealth } \\
\text { U2 }\end{array}$ \\
\hline Concomitant medications cost & $\begin{array}{l}\$ 5.64 \text { per month (artificial } \\
\text { tears) }\end{array}$ & $\begin{array}{l}\$ 10.47 \text { per month } \\
\text { (ondansetron) }\end{array}$ & $\begin{array}{l}\text { No concomitant } \\
\text { medications }\end{array}$ & $\begin{array}{l}\text { Concomitant medications from } \\
\text { DREAMM-2 for belamaf and FDA } \\
\text { label for SEL } 8,13 \\
\text { Unit costs from Red } \\
\text { Book }^{\circledR} 2019^{31}\end{array}$ \\
\hline Treatment monitoring (per month) & $\begin{array}{l}\text { Total cost: } \$ 558(C B C[\$ 44] \text {, } \\
\text { visual acuity test }[\$ 31] \text {, slit } \\
\text { lamp exam }[\$ 110] \text { and } \\
\text { physician visit }[\$ 200] \text {; all at } \\
\text { I.45 } \times \text { per month) }\end{array}$ & $\begin{array}{l}\text { Total cost: } \$ 306(\mathrm{I} \times \mathrm{CBC} \\
{[\$ 44] \text {, physician visit }[\$ 200] \text {, }} \\
\text { and blood test: chemistry } \\
\text { panel }[\$ 62])\end{array}$ & - & $\begin{array}{l}\text { Monitoring requirements from } \\
\text { GSK data on file for belamaf and } \\
\text { FDA label for } \mathrm{SEL}^{7,8} \\
\text { Unit costs from inHealth }\end{array}$ \\
\hline \multirow[t]{2}{*}{$\mathrm{AE}$ management costs } & \multicolumn{3}{|c|}{$\begin{array}{l}\text { Grade } 3-4 \text { AEs with }>5 \% \text { incidence included in the model and monthly probability } \\
\text { derived using median PFS or median treatment duration }\end{array}$} & $\begin{array}{l}\text { Incidence of Grade 3-4 AEs from } \\
\text { clinical trials }{ }^{13,22}\end{array}$ \\
\hline & $\$ 1623$ per month & $\$ 3538$ per month & Assumed no AEs & $\begin{array}{l}\text { Cost per AE episode from } \\
\text { published literature } \\
\text { and HCUP } 2016^{36} \text { (inflated to } \\
2019 \text { US\$) } \\
\text { GSK data on file for ocular AEs }\end{array}$ \\
\hline Subsequent treatment use & \multicolumn{2}{|c|}{ Subsequent treatment assumed to be BSC only for all patients } & - & Published BIM ${ }^{18}$ \\
\hline
\end{tabular}

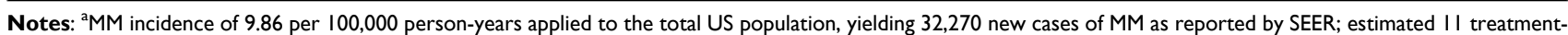
eligible patients per I million-member commercial health plan members for each model year; assumed that $100 \%$ of patients would have received a PI and immunomodulatory drug based on real-world analyses and $78.5 \%$ would have received daratumumab first to fifth line based on market share; ${ }^{b}$ Annual market share was assumed to remain constant over the model time horizon, market share of belamaf was assumed to be $50 \%$ of SEL+DEX market share in the future market mix scenario; ${ }^{\mathrm{C}}$ Estimated from median treatment duration using an exponential distribution; ${ }^{\mathrm{d} A c c o u n t s}$ for relative dose intensity, cycle length, and administration per cycle; no patient cost-sharing (co-payment or co-insurance) or rebates were assumed in the base case.

Abbreviations: AE, adverse event; belamaf, belantamab mafodotin; BIM, budget impact model; BSC, best supportive care; CBC, complete blood count; DEX, dexamethasone; FDA, US Food and Drug Administration; HCUP, Healthcare Cost and Utilization Project; MM, multiple myeloma; PI, proteasome inhibitor; PFS, progressionfree survival; Q3W, every 3 weeks; Q4W, every 4 weeks; SD, standard deviation; SEER, Surveillance, Epidemiology, and End Results; SEL, selinexor. 


\section{Model Population Inputs}

Patient mean (standard deviation [SD]) body weight was based on the belamaf $2.5 \mathrm{mg} / \mathrm{kg}$ arm of the DREAMM-2 trial. ${ }^{25}$ Given that robust epidemiology data for the target population are not available in the published literature, the estimated number of treatment-eligible patients was based on an epidemiology model. ${ }^{26}$ In brief, the epidemiology model developed was a compartmental model using differential equations to estimate patient population by LOT with 5 main LOTs (Figure 2). Additionally, the model was designed to estimate a subgroup of heavily pretreated patients with RRMM who had received $\geq 5$ prior LOTs, including a PI, an immunomodulatory agent, and an anti-CD38 mAb. The epidemiology model inputs included incidence of newly diagnosed MM, stem cell eligibility, mortality as OS, treatment duration, time to next treatment, and cytogenetic risk $^{26}$ (see Supplementary Methods). Given the US population of $327,167,434$, and 32,270 estimated new cases of MM reported by the Surveillance, Epidemiology, and End Results (SEER) cancer statistics, the MM incidence of 9.86/100,000 person-years in the US was calculated $(32,270 / 327,167,434 \times 100,000) .^{1,27}$ The epidemiology model assumed that $100 \%$ of the patients were exposed to a PI and immunomodulatory agent and $78.5 \%$ of the patients received daratumumab-based regimens across the first line to fifth line based on market share. The epidemiological model estimated a target population of 3497 treatment-eligible patients each year. ${ }^{26}$ This estimate was then divided by the US population $(327,167,434)^{27}$ and multiplied by 1 million, which equated to 11 treatmenteligible patients/per million/year in a commercial health plan. The number of treatment-eligible patients in the BIM was then distributed among the treatment options included in the analysis (belamaf, SEL+DEX, or BSC only) based on the market share estimates under the current market mix and future market mix scenarios (Figure 2).

\section{Model Cost Inputs}

Drug acquisition and administration, concomitant medications, adverse event (AE) management, treatment monitoring, and subsequent treatment costs were used to estimate total costs for each treatment under the current market mix and future market mix scenarios (the cost year was 2019).

These costs were applied over the trial-reported mean treatment duration for each regimen. At the end of the treatment duration, subsequent treatment costs were applied (a proxy measure of PFS) and patients were assumed to receive $\mathrm{BSC}$ only until the end of the model time horizon. Model inputs for cost are summarized in Table 1 and $\mathrm{AE}$ management costs in Supplementary Table S1.

\section{Model Analysis}

The budget impact was calculated as the difference in costs between the current market mix and future market mix scenarios over the model time horizon (total and annualized costs). Budgetary outcomes are expressed in terms of overall healthcare plan budget, cost per member per month (PMPM), cost per member per year (PMPY), cost per treated member (patient) per month (PTMPM), and cost per treated member per year (PTMPY) for both the current market mix and future market mix scenarios. PMPM or PMPY is the estimated cost (monthly or yearly) per member of a health plan, whereas PTMPM or PTMPY is the estimated cost (monthly or yearly) for only those members of a plan who receive treatment.

\section{Model Assumptions}

The BIM accounted only for direct treatment-related costs; non-medical direct costs and indirect costs (ie, productivity loss, personal expenditure, caregiver costs) were not included in the analysis. No rebates, co-payments, or dispensing fees are included in the current analysis. It was assumed that $100 \%$ of the target population will be treated with an active RRMM treatment as a conservative approach and that healthcare providers and patients would be $100 \%$ adherent with the recommended treatment monitoring requirements. Relative dose intensity was $82.8 \%$ for belamaf based on the pivotal clinical trial ${ }^{13}$ and was assumed to be $100 \%$ for SEL+DEX based on a publication of a BIM for SEL+DEX. ${ }^{18}$ It was also assumed that the introduction of belamaf into the market would have no impact on the disease incidence and diagnosis rate. Half of the new eligible cases of target population were assumed to start the treatment at the beginning of the budget year in which they enter the model and the other half were assumed to start the treatment at middle of the budget year, which may overestimate costs as patients become eligible for treatment throughout the year. Mean treatment duration for belamaf and SEL+DEX was calculated assuming exponential distribution based on median treatment duration observed in DREAMM-2 and STORM Part 2, ${ }^{13,22}$ respectively. Patients were assumed to permanently discontinue the active treatment for RRMM once they reach the end of treatment duration and receive BSC only until the end of the model 


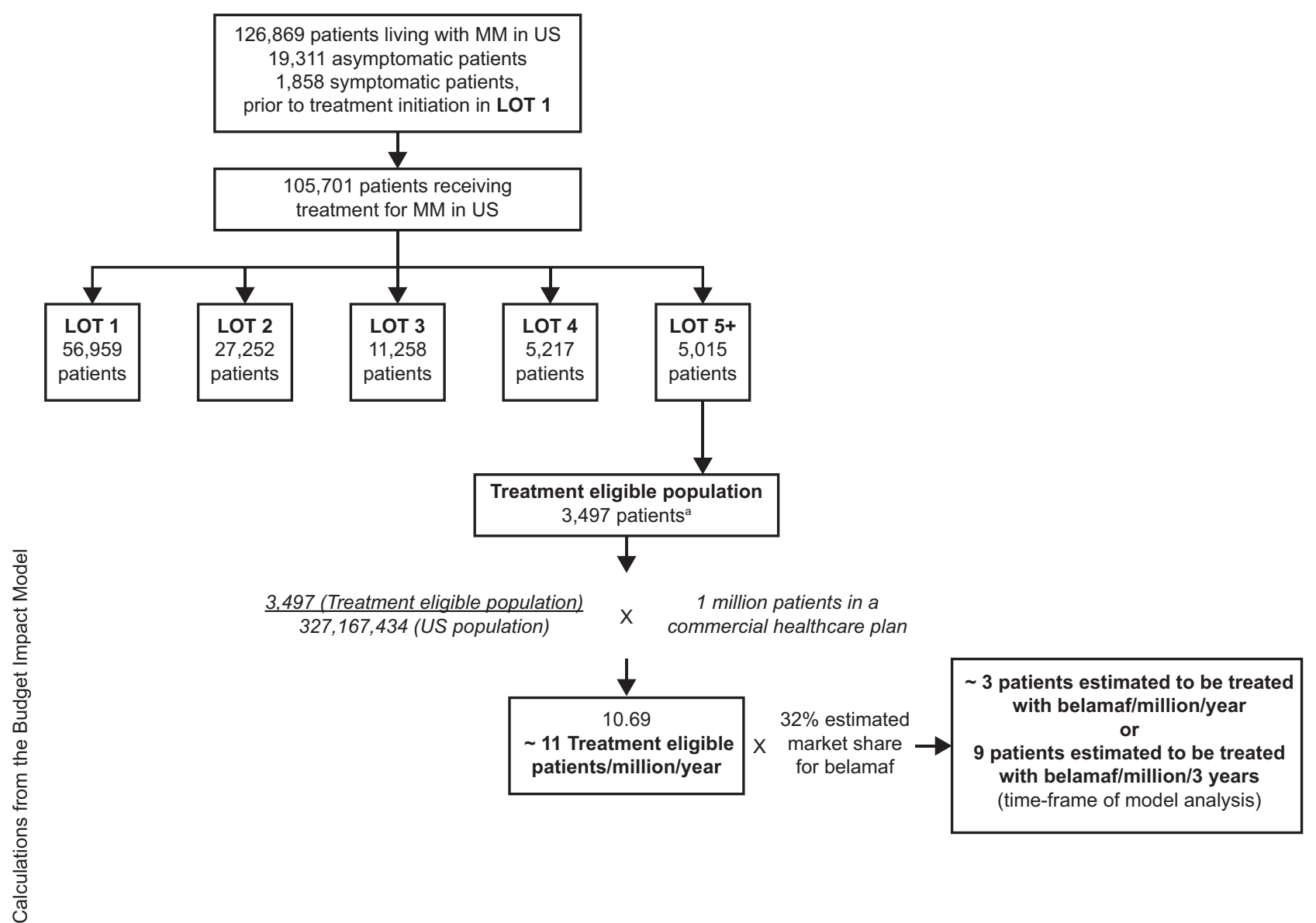

Figure 2 Steady-state patient population results from the epidemiology model, by line of therapy and showing the treatment-eligible patient population. Notes: ${ }^{a}$ Patients with prior exposure to a proteasome inhibitor, an immunomodulatory agent, and an anti-CD38 monoclonal antibody. Data from Nikolaou A, Maiese E, Samyshkin Y, et al. Estimating the Number of US Patients with Multiple Myeloma at 5 or More Lines of Treatment (LOT). International Society for Pharmacoeconomics and Outcomes Research; 16-19 November 2020, 2020; Virtual ${ }^{26}$ were used to estimate the target population for this budget impact analysis and used to calculate the number of treatment eligible patients in a commercial health plan..

Abbreviations: Belamaf, belantamab mafodotin; LOT, line of treatment; MM, multiple myeloma.

time horizon or death. The model did not account for clinical efficacy in terms of the impact of OS and PFS on the results. Model inputs related to treatment discontinuation and $\mathrm{AE}$ frequencies were assumed to match clinical practice in the United States; the costs of recognized but non-severe (Grade 1 and 2) AEs and less frequent (Grade 3-4 AEs occurring in $<5 \%$ of the patients in clinical trials) were not incorporated. Lastly, the model assumed no vial sharing for belamaf (ie, the model assumed wastage for belamaf, estimated by simulated normal distribution of body weight based on mean [SD] for patients at baseline in the $2.5 \mathrm{mg} / \mathrm{kg}$ arm of the DREAMM-2 trial).

\section{Sensitivity Analysis}

A one-way sensitivity analysis was conducted by testing the upper and lower bounds of individual model parameters to assess the impact on model outcomes. Inputs for this analysis are summarized in Supplementary Table S2.

\section{Scenario Analyses}

Scenario analyses included the use of a 1-year time horizon or a 5-year time horizon, negligible cost of mental status change $\mathrm{AE}$, and an analysis from the Medicare perspective. For the Medicare analysis, the epidemiology model population was restricted to patients age $\geq 65$ years, to estimate 30 treatment-eligible patients for each year.

Another scenario analysis was conducted to estimate the impact on the results if the number of treatmenteligible patients was based on the number of patients who received $\geq 3$ prior therapies, including an anti-CD38 $\mathrm{mAb}$, a PI and an immunomodulatory agent, as estimated from the epidemiology model. 


\section{Results}

\section{Base-Case Analysis}

The base-case analysis focused on the US budget impact of introducing belamaf to the current treatment mix for patients with RRMM who have received $\geq 4$ prior therapies, including an anti-CD38 mAb, a PI, and an immunomodulatory agent, all from the commercial payer perspective (3-year time horizon, 2019 cost year).

The inclusion of belamaf in the formulary of a hypothetical health plan for patients with RRMM resulted in a projected budget reduction of $-\$ 12,271$ over a 3-year time horizon for a 1 million-member commercial plan. The cumulative budget impact at the end of Year 3 translated to an estimated budgetneutral PMPM cost of $-\$ 0.0003$ and a change in PMPY cost of $-\$ 0.004$ (Table 2). The model estimated that within a future market scenario of 33 treatment-eligible patients, 9 of them would receive belamaf. The model assumed belamaf would displace $50 \%$ of the SEL+DEX market share but would not displace the BSC only, based on the market share assumption for BSC in the literature. ${ }^{18}$ With both the current market and future market scenarios, major cost drivers were drug acquisition, drug administration, treatment monitoring, and AE management. The largest difference in costs between the current market and future market scenarios was seen for $\mathrm{AE}$ management (reduced by $27.1 \%$ ), treatment monitoring (increased by $41.2 \%$ ), and concomitant medication (reduced by $23.1 \%)$. Savings were primarily driven by the lower $\mathrm{AE}$ management cost for Grade 3-4 AEs associated with belamaf compared with SEL+DEX. There was no difference between scenarios for subsequent treatment costs. Drug acquisition costs increased by $0.9 \%$ in the future market scenario.

\section{Sensitivity Analyses}

The results of the one-way sensitivity analysis are shown in Figure 3 and demonstrate that the cost difference in the base case was most sensitive to changes in treatment duration and drug acquisition costs. Costs of disease management after treatment discontinuation were assumed to be similar for each comparator under both the current market mix and future market mix scenarios and were therefore excluded from the analysis. With regard to subsequent treatments, a similar distribution and duration were assumed for belamaf and SEL+DEX, so cost of subsequent treatments had no impact on overall cost differences.

\section{Scenario Analyses}

The following scenarios were analyzed to understand the impact of varying key assumptions on the base-case analysis: 1-year time horizon, 5-year time horizon, negligible cost of

Table 2 Base-Case Analysis Results ${ }^{\mathrm{a}}$

\begin{tabular}{|l|l|l|l|c|}
\hline Outcomes & Current Market & Future Market & Difference (Future Market - Current Market) & \% Change \\
\hline Treatment-eligible patients, N & 33 & 33 & 0 & 0.0 \\
\hline Patients receiving belamaf, N & 0 & 9 & 9 & - \\
\hline Drug acquisition cost & $\$ 1,609,955$ & $\$ 1,624,588$ & $\$ 14,633$ & 0.9 \\
\hline Drug administration cost & $\$ 0$ & $\$ 24,280$ & $\$ 24,280$ & - \\
\hline Concomitant medication cost & $\$ 642$ & $\$ 494$ & $-\$ 148$ & -23.1 \\
\hline Treatment monitoring cost & $\$ 18,777$ & $\$ 26,509$ & $\$ 7732$ & 41.2 \\
\hline AE management cost & $\$ 217,128$ & $\$ 158,360$ & $-\$ 58,768$ & -27.1 \\
\hline Subsequent treatment cost & $\$ 213,193$ & $\$ 213,193$ & $\$ 0$ & 0.0 \\
\hline Total cost & $\$ 2,059,695$ & $\$ 2,047,424$ & $-\$ 12,271$ & -0.6 \\
\hline Total cost PMPM & $\$ 0.06$ & $\$ 0.06$ & $-\$ 0.0003$ & -0.6 \\
\hline Total cost PTMPM & $\$ 5353$ & $\$ 5321$ & $-\$ 32$ & -0.6 \\
\hline Total cost PMPY & $\$ 0.69$ & $\$ 0.68$ & $-\$ 0.004$ & -0.6 \\
\hline
\end{tabular}

Notes: ${ }^{a}$ Based on commercial payer eligible patients in a plan of I million members over time horizon ( 3 years); total costs for all three regimens (belamaf, SEL+DEX, and BSC only).

Abbreviations: AE, adverse event; belamaf, belantamab mafodotin; BSC, best supportive care; DEX, dexamethasone; PMPM, per member per month; PMPY, per member per year; PTMPM, per treated member per month; SEL, selinexor. 
mental status change AE 3-year time horizon, and Medicare perspective. Model results were robust to these scenario analyses, with a change in PMPM cost within the range of $-\$ 0.0001$ to $-\$ 0.003$, demonstrating savings in all scenarios assessed (Table 3). Another scenario analysis was conducted to estimate the impact on the results if the number of treatment-eligible patients was based on the number of patients who received $\geq 3$ prior therapies including an anti-CD38 $\mathrm{mAb}$, a PI, and an immunomodulatory agent. The introduction of belamaf for this indicated population of RRMM patients would lead to a budget impact difference of $\$ 18,093$ over a 3-year time horizon for a hypothetical 1 million-member commercial plan (Table 3, Supplementary Table S3). The cumulative budget impact at the end of Year 3 translates into a change in PMPM cost of $\$ 0.0005$ and a change in PMPY cost of $-\$ 0.01$, demonstrating minor cost savings to the commercial health plan (Table 3, Supplementary Table S4). Similar minor cost savings were observed with the Medicare perspective (PMPM of -\$0.0046 and PMPY of -\$0.05; Supplementary Table S4). Consistent with base case results, belamaf was budget neutral in patients who received $\geq 3$ prior therapies.

\section{Discussion}

Over a 3-year time horizon, the introduction of belamaf for the treatment of the indicated population of patients with RRMM (following $\geq 4$ prior therapies including an antiCD38 mAb, a PI, and an immunomodulatory agent) resulted in a net saving of $\$ 12,271$ in a 1 million-member hypothetical commercial plan, translating to a change in PMPM cost of $-\$ 0.0003$ and a change in PMPY cost of $-\$ 0.004$. The adoption of belamaf was therefore associated with a minor cost saving to the plan over 3 years.

Results of the one-way sensitivity analysis showed that this budget impact was most sensitive to changes in treatment duration and drug acquisition costs. The mean duration of treatment was assumed to be similar for belamaf and SEL + DEX based on clinical trial data, ${ }^{13,22}$ and as such was included in the model at approximately 3 months for each regimen. Monthly drug acquisition costs in the base case were slightly higher for belamaf than for SEL+DEX. The current market mix included belamaf having $0 \%$ and SEL + DEX having $64 \%$ of the market share, while the future market mix anticipated each regimen having the same $(32 \%)$ share. This would not be expected to have

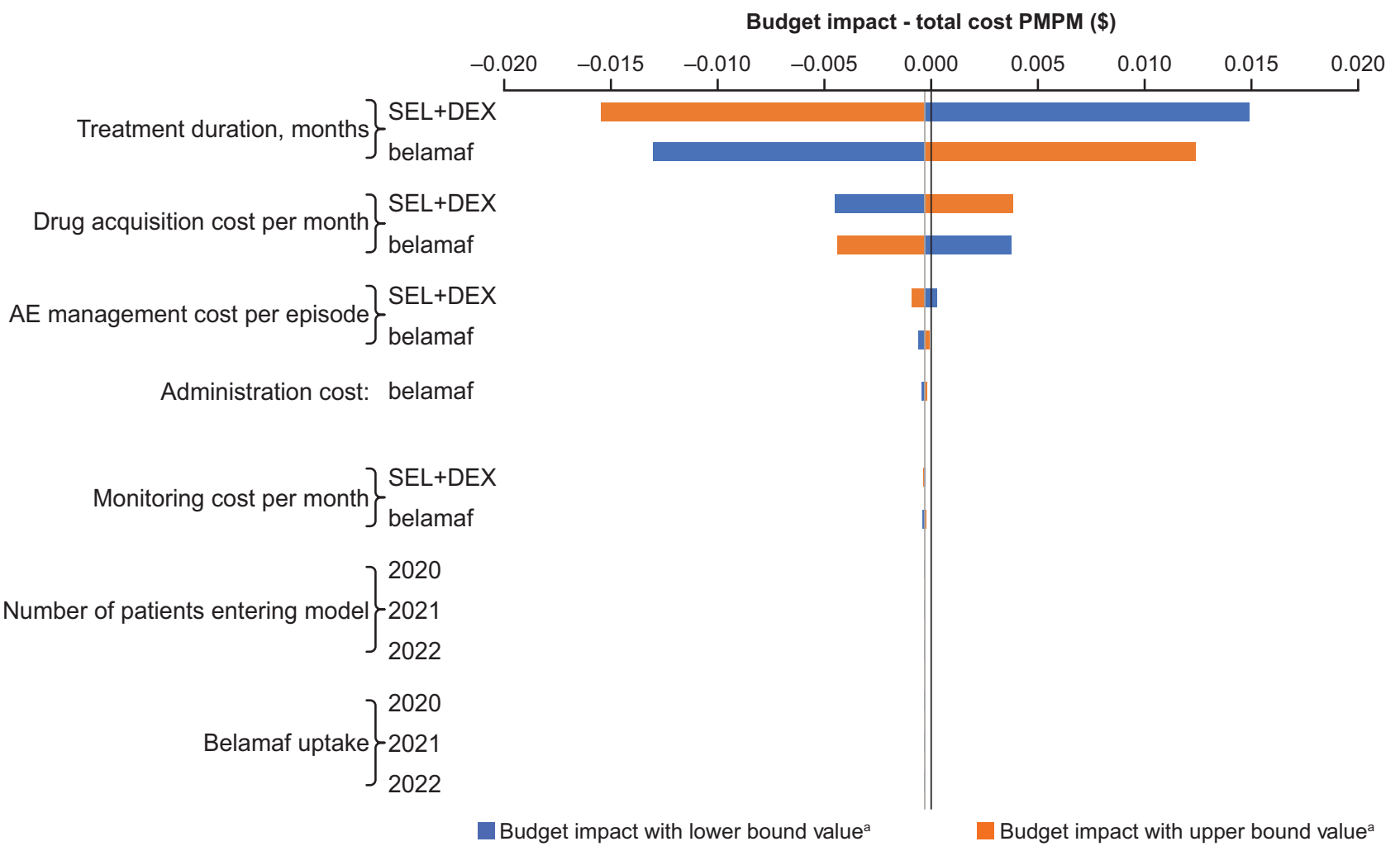

Figure 3 Results of one-way sensitivity analysis.

Notes: Base case budget impact value is $-\$ 0.0003$. ${ }^{a}$ Budget impact is in terms of incremental PMPM.

Abbreviations: AE, adverse event; belamaf, belantamab mafodotin; DEX, dexamethasone; PMPM, per member per month; SEL, selinexor. 
Table 3 Scenario Analyses Results

\begin{tabular}{|c|c|c|c|c|c|}
\hline \multirow[t]{2}{*}{ Scenario } & \multicolumn{5}{|c|}{ Change in Cost Results Over the Time Horizon } \\
\hline & Total Costs & $\begin{array}{l}\text { Total Costs } \\
\text { PMPM }\end{array}$ & $\begin{array}{l}\text { Total Costs } \\
\text { PMPY }\end{array}$ & $\begin{array}{l}\text { Total Costs } \\
\text { PTMPM }\end{array}$ & $\begin{array}{l}\text { Total Costs } \\
\text { PTMPY }\end{array}$ \\
\hline $5 \mathrm{~L}+$ base case $(3-y e a r \text { time horizon })^{a}$ & $-\$|2,27|$ & $-\$ 0.0003$ & $-\$ 0.004$ & $-\$ 32$ & $-\$ 383$ \\
\hline $5 \mathrm{~L}+\mathrm{I}$-year time horizon & $-\$ 4090.41$ & $-\$ 0.0003$ & $-\$ 0.004$ & $-\$ 31.89$ & $-\$ 382.68$ \\
\hline $5 \mathrm{~L}+5$-year time horizon & $-\$ 20,452.03$ & $-\$ 0.0003$ & $-\$ 0.004$ & $-\$ 31.89$ & $-\$ 382.68$ \\
\hline $5 \mathrm{~L}+$ Medicare perspective (3-year time horizon) ${ }^{\mathrm{b}}$ & $-\$ 98,765$ & $-\$ 0.003$ & $-\$ 0.033$ & $-\$ 91$ & $-\$ 1097$ \\
\hline $\begin{array}{l}5 \mathrm{~L}+\text { negligible cost of mental status change } \mathrm{AE} \\
\text { (3-year time horizon) }\end{array}$ & $-\$ 5285.86$ & $-\$ 0.0001$ & $-\$ 0.002$ & $-\$ 13.74$ & $-\$ 164.84$ \\
\hline $4 \mathrm{~L}+$ commercial payer (3-year time horizon $)^{c}$ & $-\$ 18,092.76$ & $-\$ 0.0005$ & $-\$ 0.01$ & $-\$ 31.89$ & $-\$ 382.68$ \\
\hline $4 \mathrm{~L}+$ Medicare perspective (3-year time horizon $)^{d}$ & $-\$ 164,814$ & $-\$ 0.0046$ & $-\$ 0.05$ & $-\$ 91.45$ & $-\$ 1097.39$ \\
\hline
\end{tabular}

Notes: ${ }^{a}$ Commercial payer perspective based on estimated treatment-eligible patient population of II patients per year in a plan of I million members; ${ }^{b}$ Estimated treatment-eligible patient population of 30 patients per year in a plan of I million members; ${ }^{\mathrm{C} C o m m e r c i a l}$ payer perspective based on estimated treatment-eligible patient population of 16 patients per year in a plan of I million members; ' ${ }^{\mathrm{E}}$ stimated treatment-eligible patient population of 50 patients per year in a plan of I million members. Abbreviations: 4L, fourth line; 5L, fifth line; AE, adverse event; PMPM, per member per month; PMPY, per member per year; PTMPM, per treated member per month; PTMPY, per treated member per year.

a substantial impact on payer budgets for the treatmenteligible heavily pretreated RRMM population (according to approved indications), since the referenced epidemiology model estimated in the US population is a relatively small group of 3497 patients who would have had prior exposure to a PI, an immunomodulatory agent, and anti-CD38 therapy. The target population estimates were based on the epidemiology model since publicly available data are outdated and do not consider specific treatment history. ${ }^{26}$

AE costs for belamaf had a negligible impact on change in PMPM cost. However, a limitation of this current analysis is that it did not include the cost of Grade 1-2 AEs, as these costs are not available in the published literature. However, the exclusion of Grade 1-2 AE cost is consistent with prior economic evaluations in RRMM. ${ }^{28,29}$ Subsequent treatment costs had no impact on cost difference results, as similar distribution and duration of subsequent treatments were assumed for all treatments, given the available data.

$\mathrm{BIM}$ results for this patient population were robust to the scenarios employing alternative time horizons (1-year and 5-year), showing that the introduction of belamaf has a similar budget impact over the short- or medium-term. The base-case results were also robust when analyzed from the perspective of a Medicare population: the small estimated budget impact of belamaf is important in this setting as the incidence of $\mathrm{MM}$ increases with age and is most frequently diagnosed among people aged $65-74$ years. ${ }^{1}$
A previous US BIA conducted in RRMM (assuming a market uptake of $64 \%$ ) showed that the introduction of selinexor was associated with a PMPM cost of $+\$ 0.0103$ in Year 3 for a hypothetical private payer plan with 4 eligible patients (out of 1 million members), based on the approved indication. ${ }^{18}$ The authors concluded that the budget impact was small and manageable. Similar to the findings of our model, the selinexor BIM results were sensitive to treatment duration and drug acquisition cost; they were also influenced by Year 1 uptake. When the base-case analysis was conducted from the Medicare perspective, the change in PMPM cost for selinexor was $+\$ 0.0078$ in Year 3 , based on 159 eligible patients. This compares with a change in PMPM cost of $-\$ 0.0003$ in Year 3 with 33 eligible patients for belamaf over 3 years in our belamaf model.

The current belamaf FDA approval is for the treatment of adults with RRMM who have received $\geq 4$ prior therapies, including an anti-CD38 mAb, a PI, and an immunomodulatory agent $^{7}$; however, data available for estimating the number of treatment-eligible patients is based on number of prior lines of treatment. Therefore, a scenario analysis was conducted to explore uncertainty regarding number of treatment-eligible patients in the model. In this scenario analysis, based on a larger pool of eligible patients $(\mathrm{N}=16$ and 50 in the commercial and Medicare plans, respectively) with RRMM who had received $\geq 3$ prior therapies including an anti-CD38 $\mathrm{mAb}$, a PI, and an immunomodulatory agent, the introduction of belamaf was found to have minor cost savings (change in PMPM cost 
of $-\$ 0.0005$ and $-\$ 0.0046$ under commercial and Medicare, respectively, over 3 years). The results in this scenario analysis were consistent with the base-case results (where belamaf was estimated to be budget neutral).

There are a number of limitations to this analysis that should be considered. The epidemiology model used to calculate the number of treatment-eligible patients in this study was based on LOTs. ${ }^{26}$ One of the limitations of the epidemiology model is predictions for the number of patients by LOT were based on steadystate results, yet in a real-world setting, these numbers would evolve with changes in the treatment landscape. ${ }^{26}$ However, it is reassuring that calculation of the number of MM patients using the model aligned with the latest SEER data. Another consideration is that half of the new belamaf-eligible cases were assumed to start the treatment at the beginning of the budget year in which they enter the model, and the other half were assumed to start the treatment in the middle of the budget year in which they enter the model. This may overestimate costs as patients become eligible for treatment throughout the year. Finally, the model relied on assumptions for future market share, post-treatment monitoring (presumed similar for all regimens), and adherence to treatment among healthcare professionals and patients (assumed to be $100 \%$ ), which, if altered, could impact the costs of management. In addition, the model does not account for the potential impact of OS and PFS on patient management costs.

Since the BIA was conducted from the payer perspective, it was restricted to direct treatment-related costs. It has been reported that treatment for RRMM also incurs indirect costs, which increase with the frequency or duration of drug administration and the number of required clinic visits. ${ }^{30}$ The results presented in this modelling study are specific to the US setting, and adaptations to other countries would necessitate the use of country-specific epidemiological data, treatment combinations, and costs.

\section{Conclusion}

This BIA indicates that if adult patients with RRMM who have received $\geq 4$ prior therapies, including an anti-CD38 $\mathrm{mAb}$, a PI, and an immunomodulatory agent, were treated with belamaf, there would be no substantial impact on a US health plan budget. Although there is a very low prevalence of this patient population in the United States, belamaf provides a novel treatment option with a new mechanism of action in these patients who have a high unmet need.

\section{Abbreviations}

AE, adverse event; belamaf, belantamab mafodotin; BIA, budget impact analysis; BIM, budget impact model; BSC, best supportive care; $\mathrm{CBC}$, complete blood count; DEX, dexamethasone; FDA, US Food and Drug Administration; HCUP, Healthcare Cost and Utilization Project; LOT, line of treatment; $\mathrm{mAb}$, monoclonal antibody; MM, multiple myeloma; ORR, overall response rate; OS, overall survival; PFS, progressionfree survival; PI, proteasome inhibitor; PMPM, per member per month; PMPY, per member per year; PTMPM, per treated member per month; PTMPY, per treated member per year; Q3W, every 3 weeks; Q4W, every 4 weeks; RRMM, relapsed/ refractory multiple myeloma; SD, standard deviation; SEER, Surveillance, Epidemiology, and End Results; SEL, selinexor.

\section{Acknowledgments}

This study was funded by GlaxoSmithKline. Medical writing support was provided by Sharon Bryant, DPT, and Crystal Kraft, $\mathrm{PhD}$, of Fishawack Indicia Ltd., part of Fishawack Health, UK, funded by GlaxoSmithKline. Drug linker technology was licensed from Seagen Inc. (Bothell, WA, USA) and the monoclonal antibody was produced with POTELLIGENT Technology licensed from BioWa (Princeton, NJ, USA).

\section{Author Contributions}

All authors contributed to data analysis, drafting or revising the article, have agreed on the journal to which the article will be submitted, gave final approval of the version to be published, and agree to be accountable for all aspects of the work.

\section{Disclosure}

AS, JCT, AA, and AN are employees of Evidera, a consulting company that received fees from GSK for work on this project. YS, BG, and FW are employees of GSK. CH and EMM were employees of GSK at the time of this analysis. The authors report no other conflicts of interest in this work.

\section{References}

1. National Institutes of Health. National Cancer Institute; Surveillance Epidemiology and End Results Program. Cancer Stat Facts: Myeloma; 2020. Available from: https://seer.cancer.gov/statfacts/html/mulmy. html. Accessed October 07, 2020.

2. Cho SF, Anderson KC, Tai YT. Targeting B Cell Maturation Antigen (BCMA) in multiple myeloma: potential uses of BCMA-based immunotherapy. Front Immunol. 2018;9:1821. doi:10.3389/ fimmu.2018.01821

3. Kumar SK, Dispenzieri A, Lacy MQ, et al. Continued improvement in survival in multiple myeloma: changes in early mortality and outcomes in older patients. Leukemia. 2014;28(5):1122-1128. doi:10.1038/leu.2013.313 
4. Rajkumar SV, Kumar S. Multiple myeloma current treatment algorithms. Blood Cancer J. 2020;10(9):94. doi:10.1038/s41408-020-00359-2

5. Verelst SGR, Blommestein HM, De Groot S, et al. Long-term outcomes in patients with multiple myeloma: a retrospective analysis of the Dutch Population-based HAematological Registry for Observational Studies (PHAROS). Hemasphere. 2018;2(4):e45. doi:10.1097/HS9.0000000000000045

6. NCCN. NCCN guidelines. Multiple myeloma. v2.2021. 2020.

7. GlaxoSmithKline. BLENREP (belantamab mafodotin-blmf) prescribing information. 2020.

8. Karyopharm Therapeutics, Inc. XPOVIO (selinexor) prescribing information. 2019.

9. Montes De Oca R, Bhattacharya S, Vitali N. The anti-BCMA antibody-drug conjugate GSK2857916 drives immunogenic cell death and immune-mediated anti-tumor responses, and in combination with an OX40 agonist potentiates in vivo activity. HemaSphere. 2019;3(S1):231. doi:10.1097/01.HS9.0000560524.63281.bc

10. Tai YT, Acharya C, An G, et al. APRIL and BCMA promote human multiple myeloma growth and immunosuppression in the bone marrow microenvironment. Blood. 2016;127(25):3225-3236. doi:10.1182/blood-2016-01-691162

11. Tai YT, Mayes PA, Acharya C, et al. Novel anti-B-cell maturation antigen antibody-drug conjugate (GSK2857916) selectively induces killing of multiple myeloma. Blood. 2014;123(20):3128-3138 doi:10.1182/blood-2013-10-535088

12. Trudel S, Lendvai N, Popat R, et al. Antibody-drug conjugate, GSK2857916, in relapsed/refractory multiple myeloma: an update on safety and efficacy from dose expansion phase I study. Blood Cancer J. 2019;9(4):37. doi:10.1038/s41408-019-0196-6

13. Lonial S, Lee HC, Badros A, et al. Belantamab mafodotin for relapsed or refractory multiple myeloma (DREAMM-2): a two-arm, randomised, open-label, Phase 2 study. Lancet Oncol. 2020;21 (2):207-221. doi:10.1016/S1470-2045(19)30788-0

14. Trudel S, Lendvai N, Popat R, et al. Targeting B-cell maturation antigen with GSK2857916 antibody-drug conjugate in relapsed or refractory multiple myeloma (BMA117159): a dose escalation and expansion phase 1 trial. Lancet Oncol. 2018;19(12):1641-1653. doi:10.1016/S1470-2045(18)30576-X

15. Lonial S, Lee HC, Badros A, et al. Longer term outcomes with singleagent belantamab mafodotin in patients with relapsed or refractory multiple myeloma: 13-month follow-up from the pivotal DREAMM-2 study. Cancer. Epub 2021 Jul 27. doi: 10.1002/cncr.33809

16. Roy A, Kish JK, Bloudek L, et al. Estimating the costs of therapy in patients with relapsed and/or refractory multiple myeloma: a model framework. Am Health Drug Benefits. 2015;8(4):204-215.

17. Fonseca R, Abouzaid S, Bonafede M, et al. Trends in overall survival and costs of multiple myeloma, 2000-2014. Leukemia. 2017;31 (9):1915-1921. doi:10.1038/leu.2016.380

18. Bassali J, Gould IG, Kaye JA, Mladsi D, Mehta J. US budget impact model for selinexor in relapsed or refractory multiple myeloma. Clinicoecon Outcomes Res. 2020;12:317-325. doi:10.2147/CEOR. S251070

19. Runyan A, Banks J, Bruni DS. Current and future oncology management in the United States. J Manag Care Spec Pharm. 2019;25 (2):272-281.

20. Sullivan SD, Mauskopf JA, Augustovski F, et al. Budget impact analysis-principles of good practice: report of the ISPOR 2012 budget impact analysis good practice II task force. Value Health. 2014;17 (1):5-14. doi:10.1016/j.jval.2013.08.2291

21. AMCP Format Executive Committee. The AMCP format for formulary submissions: welcome to version 4.0. J Manag Care Spec Pharm. 2016;22(5):444-446.

22. Chari A, Vogl DT, Gavriatopoulou M, et al. Oral selinexor-dexamethasone for triple-class refractory multiple myeloma. $N$ Engl $J$ Med. 2019;381 (8):727-738. doi:10.1056/NEJMoa1903455
23. Rakesh P, Suvannasankha A, Kapetanakis V, et al. DREAMM-2: assessing efficacy via indirect comparison of single-agent belantamab mafodotin versus selinexor plus dexamethasone combination in anti-CD38 exposed relapsed/refractory multiple myeloma. Poster presented at the 25th European Hematology Association (EHA) Congress; 2020; Virtual Format.

24. MacEwan JP, Batt K, Yin W, et al. Economic burden of multiple myeloma among patients in successive lines of therapy in the United States. Leuk Lymphoma. 2018;59(4):941-949. doi:10.1080/ 10428194.2017.1361035

25. GSK Data on File. GSK study 205678; NCT03525678.

26. Nikolaou A, Maiese E, Samyshkin Y, et al. Estimating the number of US patients with multiple myeloma at 5 or more Lines of Treatment (LOT). International Society for Pharmacoeconomics and Outcomes Research; 16-19 November, 2020; Virtual. Available from: https:// europe2020-ispor.ipostersessions.com/Default.aspx?s=79-B4-D0-A2C1-BB-30-AE-22-AA-D9-4D-BD-EF-49-8A\&pdfprint=true\&guest view $=$ true. Accessed August 31, 2021.

27. US Census Bureau. Annual estimates of the resident population for selected age groups by sex for the United States, Counties, and Puerto Rico Commonwealth and Municipios: April 1, 2010 to July 1, 2018. 2018 population estimates; 2019. Available from: https://www2.cen sus.gov/programs-surveys/popest/tables/2010-2018/counties/asrh/ PEPAGESEX.pdf. Accessed November 01, 2019.

28. Institute for Clinical and Economic Review (ICER). Treatment options for relapsed or refractory multiple myeloma: effectiveness, value, and value-based price benchmarks. Final evidence report and meeting summary; 2016. Available from: https://icerreview.org/announcements/mm-final-report/. Accessed October 07, 2020.

29. Pelligra CG, Parikh K, Guo S, et al. Cost-effectiveness of pomalidomide, carfilzomib, and daratumumab for the treatment of patients with heavily pretreated relapsed-refractory multiple myeloma in the United States. Clin Ther. 2017;39(10):1986-2005.e1985. doi:10.1016/j.clinthera.2017.08.010

30. Ailawadhi S, DerSarkissian M, Duh MS, et al. Cost offsets in the treatment journeys of patients with relapsed/refractory multiple myeloma. Clin Ther. 2019;41(3):477-493.e477. doi:10.1016/j.clinthera.2019.01.009

31. IBM Micromedex. RED BOOK $^{\circledR} 2019 ; 2019$. Available from: https://www.ibm.com/products/micromedex-red-book. Accessed October 07, 2020.

32. InHealth. Physicians' fee \& coding guide 2019. Commercial fee range - fees on disc 2019. 2019.

33. Durie B, Binder G, Pashos C, Khan Z, Hussein M, Borrello I. Total cost comparison in relapsed/refractory multiple myeloma. $J$ Med Econ. 2013;16(5):614-622. doi:10.3111/13696998.2012.760159

34. Foley KA, Wang PF, Barber BL, et al. Clinical and economic impact of infusion reactions in patients with colorectal cancer treated with cetuximab. Ann Oncol. 2010;21(7):1455-1461. doi:10.1093/annonc/ $\operatorname{mdp} 535$

35. Jakubowiak AJ, Campioni M, Benedict Á, et al. Cost-effectiveness of adding carfilzomib to lenalidomide and dexamethasone in relapsed multiple myeloma from a US perspective. J Med Econ. 2016;19 (11):1061-1074. doi:10.1080/13696998.2016.1194278

36. HCUPnet. Weighted national estimates from HCUP National Inpatient Sample (NIS), 2016, Agency for Healthcare Research and Quality (AHRQ), based on data collected by individual States and provided to AHRQ by the States. Statistics by Principal Diagnosis and Payer (insurance status); 2016. Available from: https://www. hcup-us.ahrq.gov/nisoverview.jsp. Accessed October 07, 2020. 


\section{Publish your work in this journal}

ClinicoEconomics and Outcomes Research is an international, peerreviewed open-access journal focusing on Health Technology Assessment, Pharmacoeconomics and Outcomes Research in the areas of diagnosis, medical devices, and clinical, surgical and pharmacological intervention. The economic impact of health policy and health systems organization also constitute important areas of coverage. The manuscript management system is completely online and includes a very quick and fair peer-review system, which is all easy to use. Visit http://www.dovepress.com/testimonials.php to read real quotes from published authors.

Submit your manuscript here: https://www.dovepress.com/clinicoeconomics-and-outcomes-research-journal 